\title{
Optimized local therapy for locally advanced non-small cell lung cancer
}

\author{
Charlotte Billiet ${ }^{1,2}$, Dirk De Ruysscher ${ }^{1,3}$ \\ ${ }^{1}$ Department of Radiation Oncology, KU Leuven-University of Leuven, Leuven, Belgium; ${ }^{2}$ Faculty of Medicine and Life Sciences, Hasselt \\ University, Hasselt, Belgium; ${ }^{3}$ Department of Radiation Oncology (Maastro Clinic), Maastricht University Medical Centre, GROW, Maastricht, the \\ Netherlands \\ Correspondence to: Charlotte Billiet, MD. Department of Radiation Oncology, KU Leuven-University of Leuven, Herestraat 49, 3000 Leuven, \\ Belgium. Email: charlotte.billiet@uzleuven.be or charlotte@billiet.net. \\ Provenance: This is an invited Editorial commissioned by Section Editor Dr. Jie Dai (Department of Thoracic Surgery, Shanghai Pulmonary Hospital, \\ Tongji University, Shanghai, China). \\ Comment on: Yang KL, Chang YC, Ko HL, et al. Optimizing Survival of Patients With Marginally Operable Stage IIIA Non-Small-Cell Lung Cancer \\ Receiving Chemoradiotherapy With or Without Surgery. Clin Lung Cancer 2016;17:550-7.
}

Submitted May 30, 2017. Accepted for publication Jun 01, 2017.

doi: $10.21037 /$ jtd.2017.06.77

View this article at: http://dx.doi.org/10.21037/jtd.2017.06.77

The optimal local treatment of patients with stage IIIA non-small cell lung cancer (NSCLC) is one of the most controversial areas. Specifically, the selection of patients for multimodality therapy and the sequencing of therapies remain unclear. As patients with stage IIIA disease consist of a heterogeneous group with various extents of their lung tumor, nodal status and co-morbidities, different approaches have been adopted.

Concurrent chemotherapy plus radiation therapy is considered as the standard treatment for stage IIIA NSCLC patients (1). However, a substantial portion of patients with potentially resectable disease are eligible for a multimodality approach including surgery. This was investigated in the past by different prospective randomized trials (2-5). Nevertheless, a benefit in overall survival (OS) was never demonstrated.

In the ESPATUE trial, after induction chemotherapy followed by concurrent chemoradiotherapy, resectable patients were randomized between resection and a chemoradiotherapy boost (2).

The EORTC-Lung Cancer Group randomly assigned patients to surgical resection or radiotherapy after three cycles of induction chemotherapy (3).

Albain et al. compared concurrent induction chemoradiation (cisplatin-etoposide, 45 Gy) followed by surgery versus definitive concurrent chemoradiation
(61 Gy) in potentially resectable patients (4). In this trial (INT0139), the progression-free survival (PFS) was longer in the surgical arm and a subset analysis demonstrated a better OS in the surgical patients treated with a lobectomy (vs. pneumonectomy) compared to definitive CCRT. Thus, surgical resection after induction treatment seems an option in a selection of stage IIIA NSCLC patients.

Induction chemotherapy was compared with induction chemoradiotherapy followed by surgery by the Swiss Group for Clinical Cancer Research (5). No significant benefit in OS or event-free-survival was reported. Therefore, they concluded that one definitive local treatment modality combined with neoadjuvant chemotherapy was sufficient. Although we have to remark that all three treatment modalities are required in an attempt to maximize the local control probability, for example in pancoast tumors.

However, the upfront selection of patients for a surgical resection is challenging, due to both patient (comorbidity, refusal) and tumor (no downsizing or downstaging) related factors. Unfortunately, this may lead to incomplete resections (R1/2), which is a known negative prognostic factor $(6,7)$ and a higher rate of pneumonectomy, which are associated with higher mortality and morbidity rates (4).

Therefore, Yang et al. conducted an interesting retrospective study with a phased chemoradiotherapy protocol in 47 patients (8). After neoadjuvant CCRT 
(36-50 Gy plus concurrent cisplatin and vinorelbine), the operability was reassessed. If surgery was not possible, due to patient (comorbidity, refusal) or tumor (inoperability) related factors, docetaxel-based maintenance chemotherapy was given, followed by a split-course chemoradiotherapy boost (20 to 30 Gy plus concurrent vinorelbine). A third group of patients received continuous-course definitive CCRT. They hypothesized that the OS rates could be optimized by maximizing the probability of patients for surgery and the effect of docetaxel-based maintenance chemotherapy on decreasing the tumor size, allowing adequate split-course boost in the situation that no surgery was chosen.

The findings of this study may suggest a significant better OS and a similar PFS in the phased protocol (surgery or split-course CCRT boost) compared to the definitive chemoradiation group. This OS improvement was contributed to a better local control with addition of surgery, a suppressing of the tumor in the reassessed inoperable patients with maintenance therapy and a limited toxicity [absence of $\geq$ grade 3 radiation pneumonitis (RP)] in the phased protocol patients. In the surgery group, no patients received a pneumonectomy; the completeness of resection was not reported.

We believe that proper patient selection and a tailored approach is mandatory in the multimodality treatment for stage IIIA NSCLC patients. This can be achieved by a more accurate staging: With recent developments in staging techniques, pre-treatment PET-CT scan, brain imaging and minimal invasive mediastinal staging with endobronchial ultrasound (EBUS) or esophageal ultrasound are now uses as standard staging methods. Yang et al. (8) not reported which staging investigations were used, however, as patients were included between 2006 and 2013, these techniques were already introduced in this time period.

Offering this split-course treatment with maintenance chemotherapy might indeed be beneficial to increase the number of patients treated with a surgical multimodality approach. However, we can argue if this phased chemoradiation is really necessary? There is no demonstrated benefit regarding the use of neo-adjuvant chemoradiotherapy compared to chemotherapy alone $(5,9)$ in a surgical multimodality approach. Considering this, it can be sufficient to treat with induction chemotherapy alone, followed by a reassessment of the operability. In this case, the disadvantages of performing surgery in irradiated tissue (increased morbidity, higher risk of wound healing problems, etc.) can be neglected. Moreover, the restaging will be facilitated.

After a reassessment, the patient can be deemed operable in case of a good response on induction therapy. Depending on the pathology report, an additional radiation treatment will be mandatory. We earlier demonstrated the possible benefit and safety of postoperative radiotherapy (PORT) in stage III NSCLC patients with persistent nodal involvement (ypN2 status) and/or incomplete resection (R1/2 resection) (10). PORT was investigated using the current more modern treatment and staging strategies in contrast with older, suboptimal techniques.

Besides the issues concerning this split-course treatment, questions arises about maximizing the number of surgically treated patients. Yang et al. demonstrated an excellent OS in the surgical group (2-year OS $=90.0 \%$ ) and contributed this to a better local control with surgery. However, we believe that this surgical group is a positive selection of patients: fit patients with good performance status and tumors with a good response after induction therapy (locoregional and subclinical metastatic disease). So it is not surprisingly that higher OS rates were seen in this group. Because of some major limitations of this study, it is difficult to draw conclusions about the superiority of a surgical multimodality treatment. First, there is this selection bias, because of the non-randomized character of this study, as described above. Second, this is a small and retrospective study, as reported by the authors, prospective studies are needed to confirm the efficacy and safety of this phased CCRT protocol.

Currently, there is no evidence of the superiority of a surgical combined treatment, compared with definitive chemoradiation (2-5). Definitive chemoradiotherapy can be offered, rather concurrent than sequential (11), with radiation doses up to $60 \mathrm{~Gy}$. Both are acceptable strategies for this good-prognosis stage III-N2 patient group.

What about toxicity concerns? In the phased protocol group of this study, the radiation toxicity was acceptable with a non-significantly lower probability of symptomatic RP (<20\%) compared to the continuous CCRT group. However, the toxicity of the definitive CCRT treatment was also limited in this study, with only $5.3 \%$ of patients experiencing grade 3 toxicity. We earlier investigated toxicity in surgically treated stage III NSCLC patients and found no significant difference in cancer-related deaths and cardiac or pulmonary toxicity between an irradiated subgroup and a non-irradiated subgroup (10). We therefore concluded that the toxicity profile of PORT is acceptable. Other factors, such as the upfront cardiac and pulmonary comorbidity and the major surgery, could probably explain 
the cardiac and pulmonary events during follow-up.

In conclusion, both trimodality treatment that include surgery and bimodality treatment without surgery but with a definitive chemoradiotherapy boost, lead to longterm OS and PFS, with 5-year OS rates around 30-40\%, and an acceptable toxicity profile using modern treatment and staging strategies. We believe that a tailored approach with proper patient selection can be more appropriate than offering induction chemoradiotherapy in all cases.

\section{Acknowledgements}

This work was supported by the foundation Limburg Sterk Merk, province of Limburg, Flemish government, Hasselt University, Ziekenhuis Oost-Limburg and Jessa Hospital. Charlotte Billiet was supported by the Limburg Cancer Foundation and Kom op tegen kanker.

\section{Footnote}

Conflicts of Interest: The authors have no conflicts of interest to declare.

\section{References}

1. Eberhardt WE, De Ruysscher D, Weder W, et al. 2nd ESMO Consensus Conference in Lung Cancer: locally advanced stage III non-small-cell lung cancer. Ann Oncol 2015;26:1573-88.

2. Eberhardt WEE, Pöttgen C, Gauler TC, et al. Phase III Study of Surgery Versus Definitive Concurrent Chemoradiotherapy Boost in Patients With Resectable Stage IIIA(N2) and Selected IIIB Non-Small-Cell Lung Cancer After Induction Chemotherapy and Concurrent Chemoradiotherapy (ESPATUE). J Clin Oncol 2015;33:4194-201.

3. van Meerbeeck JP, Kramer GW, Van Schil PE, et al. Randomized controlled trial of resection versus

Cite this article as: Billiet C, De Ruysscher D. Optimized local therapy for locally advanced non-small cell lung cancer. J Thorac Dis 2017;9(7):1783-1785. doi: 10.21037/jtd.2017.06.77 radiotherapy after induction chemotherapy in stage IIIA-N2 non-small-cell lung cancer. J Natl Cancer Inst 2007;99:442-50.

4. Albain KS, Swann RS, Rusch VR, et al. Phase III study of concurrent chemotherapy and full course radiotherapy (CT/RT) versus CT/RT followed by surgical resection for stage IIIA(pN2) non-small cell lung cancer (NSCLC): outcomes update of North American Intergroup 0139 (RTOG 9309). Lancet 2009;374:379-86.

5. Pless M, Stupp R, Ris HB, et al. Induction chemoradiation in stage IIIA/N2 non-small-cell lung cancer: a phase 3 randomised trial. Lancet 2015;386:1049-56.

6. Betticher DC, Hsu Schmitz SF, Tötsch M, et al. Mediastinal lymph node clearance after docetaxel-cisplatin neoadjuvant chemotherapy is prognostic of survival in patients with stage IIIA pN2 non-small-cell lung cancer: a multicenter phase II trial. J Clin Oncol 2003;21:1752-9.

7. Martini N, Kris MG, Flehinger BJ, et al. Preoperative chemotherapy for stage IIIa (N2) lung cancer: the SloanKettering experience with 136 patients. Ann Thorac Surg 1993;55:1365-73;discussion 1373-4.

8. Yang KL, Chang YC, Ko HL, et al. Optimizing Survival of Patients With Marginally Operable Stage IIIA Non-SmallCell Lung Cancer Receiving Chemoradiotherapy With or Without Surgery. Clin Lung Cancer 2016;17:550-7.

9. Thomas M, Rübe C, Hoffknecht P, et al. Effect of preoperative chemoradiation in addition to preoperative chemotherapy: a randomised trial in stage III non-smallcell lung cancer. Lancet Oncol 2008;9:636-48.

10. Billiet C, Peeters S, Decaluwé H, et al. 126P: Outcome after post-operative radiotherapy (PORT) in ypN2 or R1/ $\mathrm{R} 2$ versus no PORT in ypN0 stage III-N2 non-small cell lung cancer after induction chemotherapy and resection. J Thorac Oncol 2016;11:S110-1.

11. Aupérin A, Le Péchoux C, Rolland E, et al. Meta-analysis of concomitant versus sequential radiochemotherapy in locally advanced non-small-cell lung cancer. J Clin Oncol 2010;28:2181-90. 